\title{
Optical spectrometer at the nanoscale using optical Yagi-Uda nanoantennas
}

\author{
Jingjing Li, Alessandro Salandrino, and Nader Engheta* \\ Department of Electrical and Systems Engineering, University of Pennsylvania, Philadelphia, Pennsylvania 19104, USA
}

(Received 17 July 2008; revised manuscript received 4 January 2009; published 5 May 2009)

\begin{abstract}
Here we present and analyze an optical spectrum analyzer at the nanometer scale that is able to distribute different frequency contents of the radiation of an optical dipole source into different directions in the space. The spectrum analyzer is composed of arrays of optical Yagi-Uda nanoantennas, forming relatively narrow radiation patterns operating at different frequencies. The optical Yagi-Uda nanoantennas composed of plasmonic core-shell nanoparticles are used as an example of building blocks for this idea in our study. Narrow radiation beams in such antenna arrays are realized by tailoring the scattering phase of the nanoparticles. The sensitivity of such an antenna array to the operating wavelength and the angular distribution of the radiation pattern, which is essential for the operation of the spectrum analyzer proposed here, is studied theoretically. The chromatic dispersion and the angular variation of the radiation pattern of such an optical spectrum analyzer are discussed in detail.
\end{abstract}

DOI: 10.1103/PhysRevB.79.195104

PACS number(s): 73.20.Mf, 84.40.Ba, 07.60.Rd, 78.67.Bf

\section{INTRODUCTION}

Design and fabrication of antenna devices that receive and transmit optical signals coherently have gained growing interests in the recent years. ${ }^{1-4}$ Since metals no longer possess high conductivity in the optical domain, but rather they are described as materials whose relative permittivities show negative real parts (plasmonic materials), their interaction with electromagnetic wave at optical frequencies is significantly different from that in microwave and radio frequency (RF) domains. Therefore, the conventional antenna design techniques maturely developed at microwave or RF frequencies need to be revised properly for optical wavelengths, which makes the design of optical antenna a challenging task. There have been several optical antennas proposed by exploiting the plasmonic features of metals in the optical regime. The subwavelength plasmonic particles near their scattering resonance are of special interests for design of optical antennas. Near the scattering resonance, these particles can be described as induced dipoles with large polarizabilities. The interaction of the particles with the electromagnetic wave is then relatively straightforward to describe, making it a useful approach for optical antenna design. For example, in one of our earlier works we have presented a design of optical antenna system with feeding mechanism included by placing plasmonic particles near a slab waveguide. ${ }^{4}$ Also, in our group we have explored the concept of optical input impedance for optical nanoantennas, providing a powerful tool for tuning and designing optical antennas at desired wavelengths. ${ }^{5,6}$

There have been extensive studies on the influence of the environment to the emission of a stimulated fluorescent molecule. Different situations such as a molecule above a semiinfinite substrate ${ }^{7,8}$ or stratified layers, ${ }^{9,10}$ a molecule near a spherical or ellipsoidal particle, ${ }^{11}$ or a molecule inside a dielectric sphere were discussed. ${ }^{12}$ The existence of plasmonic objects may have prominent influence on the molecular fluorescence, owing to the coupling between the field radiated from the molecule and the plasmon polaritons. ${ }^{13,14}$ In these studies, the molecule is modeled as a damped electric dipole and the influence from the outside environment is ordinarily described in terms of life time and/or quantum efficiency change, etc. It is not until very recently when attention has been paid by various groups to the wavelength sensitivity and spatial reshaping of the radiation patterns in different environments and material backgrounds. ${ }^{15-20}$ In the present study, motivated by one of our earlier studies on Yagi-Udatype optical antennas, ${ }^{21}$ we discuss a method of controlling the angular distribution of the optical dipole radiation (e.g., from a quantum dot or from a fluorescent molecule) using such nanoparticle-based Yagi-Uda optical antennas, with potential applications in molecular spectroscopy.

The current discussion is based on the optical Yagi-Uda nanoantenna we proposed in Ref. 21. Such an optical nanoantenna is composed of several subwavelength-sized plasmonic nanoparticles and is driven by an optical dipole source. The particles are modeled as induced dipoles that are coupled to each other and to the source, and the magnitude and the shape of the radiation pattern of the dipole source can be tailored when resonant plasmonic nanoparticles with large polarizabilities are used. Core-shell plasmonic nanoparticles with the core made of an ordinary dielectric and the shell of a plasmonic material, or vice versa, are used in this system (Fig. 1(a)) since their resonant frequencies can be adjusted in a broad range by varying the ratio of the core radius $(b)$ to the outer radius $(a) .^{22}$ Specifically, $b / a$ of each particle can be designed deliberately to "detune" its resonance in order to achieve a phase of induced dipole less than or greater than $\pi / 2$ with respect to the phase of the incident field [refer to Fig. 1(a)], so that they may play the role of the "reflectors" or the "directors" in a conventional RF Yagi-Uda antenna. ${ }^{23,24}$ The radiation pattern of such an optical antenna array then exhibits a relatively narrow beam toward the direction of the "directors" and a minimum (or a null) toward the direction of the reflector, analogous to the RF Yagi-Uda antennas. Such an optical nanoantenna is used as an example of a building block to construct the concept of an optical spectrum analyzer, as we discuss below. 

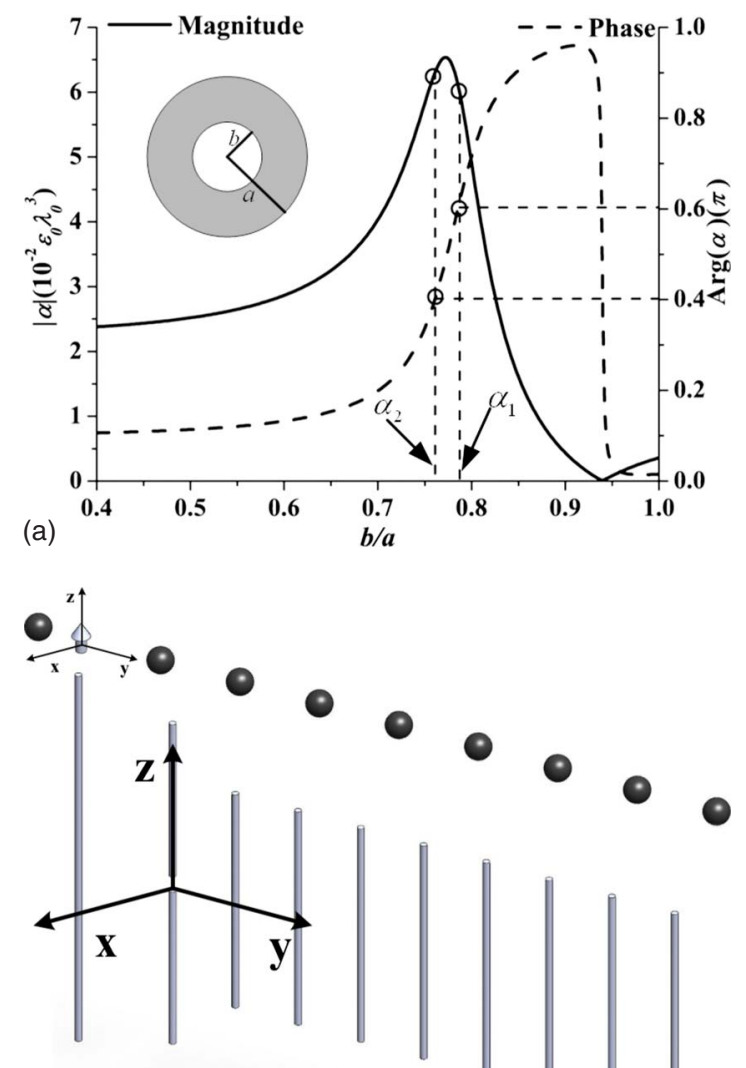

(b)

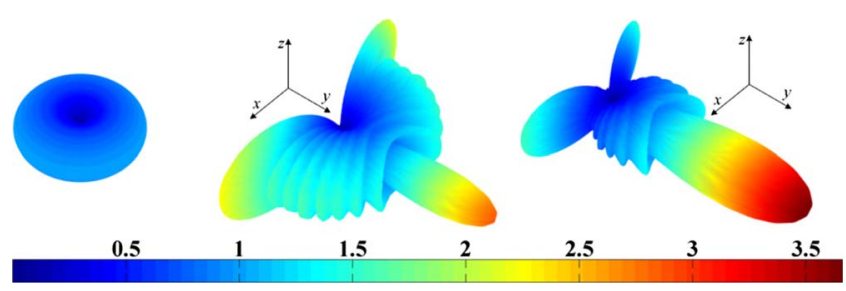

(c)

FIG. 1. (Color online) (a) Magnitude and phase of the polarizability $\alpha$ vs $b / a$ of a concentric core-shell particle [core: $\mathrm{SiO}_{2}$ with $\varepsilon_{1}=2.2 \varepsilon_{0}$, shell: silver with $\left.\varepsilon_{2}=(-15.33+0.451 i) \varepsilon_{0}\right]$ at $\lambda_{0}$ $=517 \mathrm{~nm}$. The marked points are the parameters with phase of $0.4 \pi$ and $0.6 \pi$. (b) The diagram of a nine-particle optical Yagi-Uda nanoantenna and a classical Yagi-Uda antenna in the RF and microwave domain that is composed of same number of linear antennas. (c) The 3D power-density patterns of the source dipole when radiating alone (left panel) and that of the optical Yagi-Uda antenna at $580 \mathrm{THz}(517 \mathrm{~nm})$ (middle panel) and $559 \mathrm{THz}(537 \mathrm{~nm})$ (right panel). Patterns are normalized to the maximum power density of the dipole when radiating alone.

\section{WAVELENGTH SENSITIVITY IN OPTICAL YAGI-UDA NANOANTENNAS}

The geometry of an example of optical Yagi-Uda nanoantenna is given in Fig. 1(b), together with a conventional RF Yagi-Uda antenna array. This optical antenna is designed to operate at $580 \mathrm{THz}$ or $\lambda_{0}=517 \mathrm{~nm}$. One reflector and eight directors are used in this design, and the core-shell particles are assumed to be made of $\mathrm{SiO}_{2}$ (for the core) and silver (for the shell), with $0.1 \lambda_{0}$ outer radius. For the particle at the left

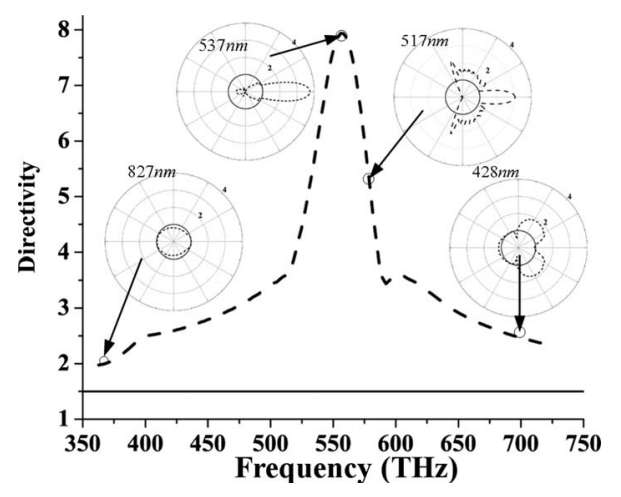

FIG. 2. The directivity (dashed line) of the optical Yagi-Uda nanoantenna in Fig. 1(b). Solid line: The directivity of the dipole source when radiating alone. Insets: the $H$-plane power pattern of the Yagi-Uda antenna at different operating wavelengths, in which the dashed line is the pattern of the antenna while the solid line is that of the dipole alone.

we choose $b / a=0.785$ so that $\alpha_{1}$ has a phase of $0.6 \pi$ (the so-called reflector), while each of the eight nanoparticles on right side has $b / a=0.761$ so that the phase of $\alpha_{2}$ is $0.4 \pi$ (the directors) [see the lines and marks in Fig. 1(a)]. The reflector-source distance is $d_{1}=0.25 \lambda_{0}$, and the distance between every two neighboring directors on the right is $d_{2}$ $=0.72 \lambda_{0}$, which is the same as the distance between the dipole source and the first director. The material loss in silver is taken into account by using realistic material parameters reported from experimental studies in the literature (e.g., Ref. 25). The three-dimensional (3D) optical radiation pattern of such an optical nanoantenna at $517 \mathrm{~nm}$ wavelength is shown in the middle panel of Fig. 1(c) with a narrow beam pointing to $\hat{\mathbf{y}}$ direction and a much smaller radiation to the opposite direction.

Similar to many other antenna systems, the beam pattern of an optical Yagi-Uda nanoantennas is sensitive to the variation of the operating wavelength for at least two reasons: (1) the relative permittivities of the plasmonic materials forming the nanoparticles, and the resulting electric polarizability of these particles, are wavelength dependent; (2) when the sizes of the nanoparticles and their relative positions are decided and then kept fixed, different operating wavelengths would lead to different relative electrical sizes and relative distances, which causes variation in coupling among particles. Therefore, optical nanoantennas composed of plasmonic particles are intrinsically sensitive to wavelength variation. To examine the frequency dispersion of the optical Yagi-Uda nanoantenna shown in Fig. 1(b), we look at the directivity, $D$, of the antenna, defined as $D=4 \pi U / P_{\text {rad }}$, where $U$ is the maximum radiation intensity, i.e., the intensity of the main beam (W/unit solid angle) and $P_{\text {rad }}$ is the total radiated power. The result is shown in Fig. 2 together with power patterns in the $H$ plane $[x-y$ plane, refer to Fig. $1(\mathrm{~b})]$ at several typical operating wavelengths. Each pattern is normalized with respect to the maximum power flux density (i.e., the intensity of the main beam) of the dipole source if the dipole radiates alone (i.e., in the absence of any particles) at that operating wavelength (keeping the same dipole moment $p)$. As can be seen, the directivity has a sharp peak near the 
design wavelength. The maximum directivity is 7.97 , higher than that of the radiating dipole source alone, which is 1.5 and shown as the solid black line. Away from the design wavelength, for example, at $363 \mathrm{THz}(827 \mathrm{~nm})$ or $701 \mathrm{THz}$ $(428 \mathrm{~nm})$, the directivity gets worse and the pattern is closer to that of the dipole source alone (see the insets in Fig. 2). It is interesting to point out that although the antenna was originally designed to work at $580 \mathrm{THz}(517 \mathrm{~nm})$, the maximum directivity is actually achieved at a slightly lower frequency $559 \mathrm{THz}(537 \mathrm{~nm})$ and the system exhibits a sharper beam at this operating wavelength as shown by the inset in Fig. 2. For the sake of comparison, the 3D power pattern at this wavelength is also shown in Fig. 1(c) (the right panel). At this operating wavelength, the phase of the polarizability of the reflector nanoparticle is $0.45 \pi$ and that of each of the eight director particles is $0.30 \pi$. As far as the directivity is concerned, such a pattern is more desirable than the one at $580 \mathrm{THz}(517 \mathrm{~nm})$.

\section{NANOSCALE SPECTRUM ANALYZER AT OPTICAL FREQUENCIES}

From the above discussion, it is evident that optical YagiUda nanoantenna can be considered as a selective system for wavelength sensitivity and angular variation. Innovative nanoscale devices can therefore be envisioned based on such selectivity. For instance, a set of several optical Yagi-Uda nanoantennas may be designed such that each is to work at a different wavelength with its maximum beam pointing to a different direction. When driving by a broad-band dipole source whose emission spectrum covers a wide range of wavelengths (which is typical for a florescent molecule), radiations at selected wavelengths may be distributed into selected directions. As a design example, we consider a system of two Yagi-Uda optical nanoantennas. Each one has eight directors and is designed following the procedure described in detail in Ref. 21 using concentric core-shell plasmonic particles with the core made of $\mathrm{SiO}_{2}$ and the shell of silver. The first Yagi-Uda (YU1) is the one shown in Fig. 1(a) and is designed to work at $558 \mathrm{THz}\left(\lambda_{0}=537 \mathrm{~nm}\right)$. Its pattern at this wavelength is shown in the right panel in Fig. 1(c). The other one (YU2) is designed to work at $464 \mathrm{THz}\left(\lambda_{0}\right.$ $=646 \mathrm{~nm}$ ). The radiated power pattern of YU2 at $646 \mathrm{~nm}$ is similar to that of YU1 at $558 \mathrm{THz}(537 \mathrm{~nm})$. Of course, the ranges of the operating wavelengths of YU1 and YU2 are different (see the directivity plot of YU1 in Fig. 2). YU1 has a directivity peak at $558 \mathrm{THz}(537 \mathrm{~nm})$ while YU2 has a peak at $464.1 \mathrm{THz}(646 \mathrm{~nm})$. The two Yagi-Uda optical antennas are then put together such that they are both driven by the same dipole source, as shown in Fig. 3(a). In this plot, the eight particles at the $-\hat{\mathbf{y}}$ axis (only four of them are shown as light-colored spheres) are the directors of YU2, while the light-colored one at the $+\hat{\mathbf{y}}$ side of the dipole source (shown as an arrow) is its reflector. Of course, the maximum beam of YU2 is designed to point to the $-\hat{\mathbf{y}}$. The other particles (shown as dark-colored ones) are those of YU1, whose maximum beam is arranged to point to the $+\hat{\mathbf{y}}$ direction.

When calculating the radiation properties of such a system at any wavelength, the influence of, and coupling

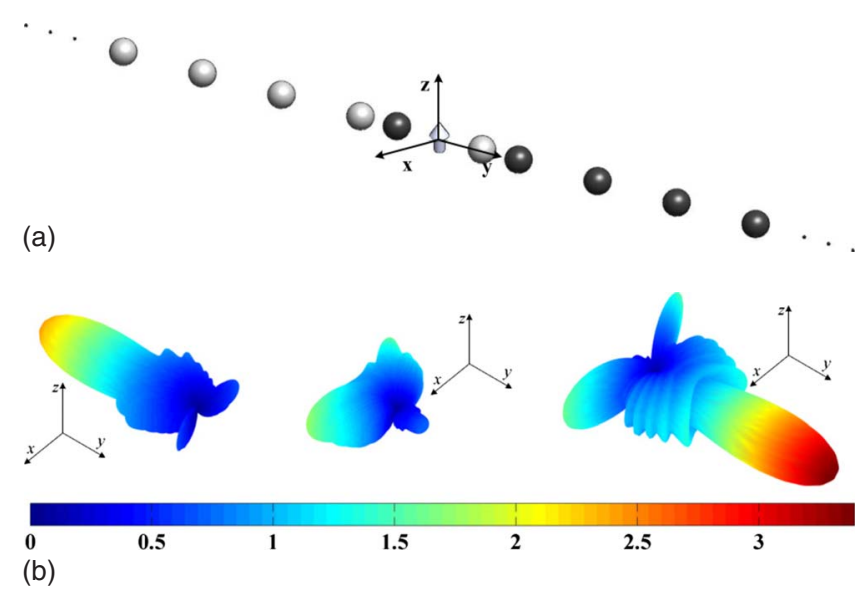

FIG. 3. (Color online) Design of an optical nanoscale spectrum analyzer composed of two Yagi-Uda optical antennas. (a) The geometry of the system. (b) The radiated power patterns of the system at different operating wavelengths. Left: at $464 \mathrm{THz}(646 \mathrm{~nm})$; middle: at $481 \mathrm{THz}(623 \mathrm{~nm})$; right: at $561 \mathrm{THz}(534 \mathrm{~nm})$.

among, all the 18 particles and the original dipole source are included. The radiated power patterns at different wavelengths are shown in Fig. 3(b). We notice that at $561 \mathrm{THz}$ $(534 \mathrm{~nm})$, the pattern [the right panel in Fig. 3(b)] shows a narrow beam pointing toward the $+\hat{\mathbf{y}}$ direction. Such a pattern is similar to that of YU1 at the same operating wavelength, as seen in Fig. 1(c). Actually, at this operating wavelength YU2 has little influence on the dipole source and YU1 dominates the behavior of the entire system. As the operating frequency (wavelength) changes to $464 \mathrm{THz}(646 \mathrm{~nm})$, YU2 dominates and the pattern [the left one in Fig. 3(b)] shows a narrow beam pointing to $-\hat{y}$ direction, which looks similar to that of YU2 at this frequency (not shown here). At the operating wavelengths in between, such as $474 \mathrm{THz}(623 \mathrm{~nm})$ at which the patterns are shown in the middle panel in Fig. 3 (b), the radiation magnitude is much smaller and the pattern does not show any noticeable increase in directivity-very different from those at $464 \mathrm{THz}(646 \mathrm{~nm})$ or $561 \mathrm{THz}(534$ $\mathrm{nm}$ ). The calculated directivity of the whole system is shown in Fig. 4(a). As expected, the plot shows two peaks at the operating frequency (wavelength) of $464 \mathrm{THz}(646 \mathrm{~nm})(\mathrm{ob}-$ served at $-\hat{\mathbf{y}})$ and $561 \mathrm{THz}(534 \mathrm{~nm})($ observed at $+\hat{\mathbf{y}})$, respectively. Clearly, the radiation at these two different wavelengths is distributed to two different directions by this system.

An interesting feature of the system is the spectrum an observer can record when sitting at a specific observation angle. Figure 4(b) shows the calculated far-zone radiation intensity vs operating wavelength when the observer is at different positions in the $H$ plane $[x-y$ plane in this case, refer to Fig. 3(a)]. The dashed line (green online) is the spectrum observed at $+\hat{\mathbf{y}}$, while the solid line (red online) is that at $-\hat{\mathbf{y}}$, respectively. The calculation is performed under the assumption that the wide-band dipole source gives out the uniform total power when radiating alone (i.e., in the absence of any particle) at different wavelengths. In other words, for the sake of simplicity and to highlight the role of frequency dispersion of the collections of nanoparticles only, the emission 

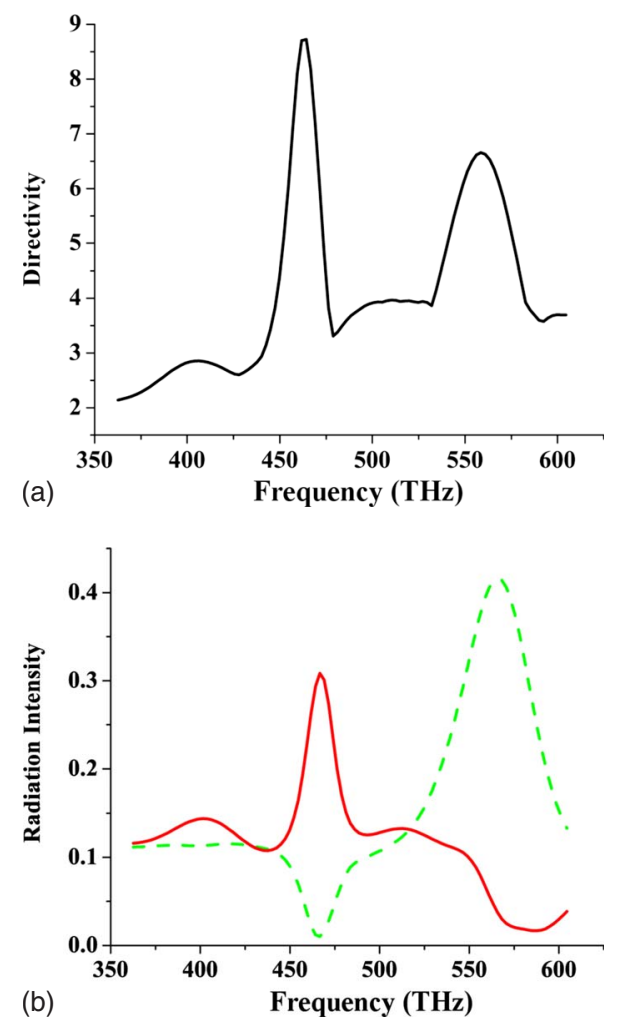

FIG. 4. (Color online) (a) The directivity of the spectrum analyzer shown in Fig. 3(a). (b) The radiation intensity of the spectrum analyzer observed from $-\hat{\mathbf{y}}$ direction (the solid line, red online) or from the $+\hat{\mathbf{y}}$ direction (the dashed line, green online), normalized to the total radiated power of the source dipole when radiating alone.

spectrum of the dipole source is assumed to be flat over the wavelength band. When observing at the $+\hat{\mathbf{y}}$ side, the radiation intensity is maximized around $566 \mathrm{THz}(530 \mathrm{~nm}$, green color, dashed line), while at around $467 \mathrm{THz}(642 \mathrm{~nm}$ or red color, solid line) the radiation intensity is minimized. On the other hand, the radiation intensity observed at $-\hat{\mathbf{y}}$ side is almost the complement, which is maximized at $467 \mathrm{THz}$ $(642 \mathrm{~nm})$ for red color and minimized around $587.6 \mathrm{THz}$
$(510 \mathrm{~nm})$ for green color. Therefore, if we observe from the $+\hat{\mathbf{y}}$ side, we will mostly see a green color, while from $-\hat{\mathbf{y}}$ side a red color is seen, although the same source is used. If the emission spectrum of the dipole source (i.e., fluorescent molecule) itself possesses any specific dispersion, the received spectrum would be the spectrum shown in Fig. 4(b) multiplied by the intrinsic emission spectrum. Such a system therefore maps the spectral information of the fluorescent molecule into the (spatial) angular domain. In this way, this nanodevice essentially operates as a nanoscale spectrum analyzer at optical wavelengths.

In the above design of the nanoscale optical spectrometer the two Yagi-Uda nanoantennas are arranged collinearly (i.e., back to back with all particles aligned). Such a collinear arrangement makes the best use of the directivity separation of the two Yagi-Uda antennas such that the spectra recorded at the two directions $(+\hat{\mathbf{y}}$ and $-\hat{\mathbf{y}})$ are mostly different. Another possibility would be to have the two (or more) YagiUda antennas arranged with a given angular separation with each other, as shown in Fig. 5(a). One of the Yagi-Uda antennas (YU2) is optimized at the absorption wavelength of the dipole source (e.g., molecule) with its main beam pointing toward the direction of $\hat{x}$, while the other (YU1) is optimized at the emission wavelength and its main beam points to an angle, e.g., $120^{\circ}$ from $\hat{x}$. Here YU1 and YU2 are similar to those in Fig. 1. Under this arrangement, YU2 can work under "receiving" (i.e., absorption) mode at the absorption wavelength such that the molecule can effectively absorb the incoming radiation along $\hat{x}$, its main axis, while YU1 will distribute the fluorescent emission of the molecule toward its main axis. The directivity of this system is shown in Fig. 5(b). Again, two peaks are observed, with the one at $556 \mathrm{THz}$ $(539 \mathrm{~nm})$ corresponding to the maximum reception from incoming energy along $\hat{x}$ and the one at $459 \mathrm{THz}(653 \mathrm{~nm})$ corresponding to the sharp radiation beam toward the other direction. The patterns are shown in Fig. 5(c).

\section{DISCUSSIONS AND CONCLUSIONS}

Using the nanoscale spectrum analyzer proposed in this paper, the absorption and emission of a fluorescent molecule

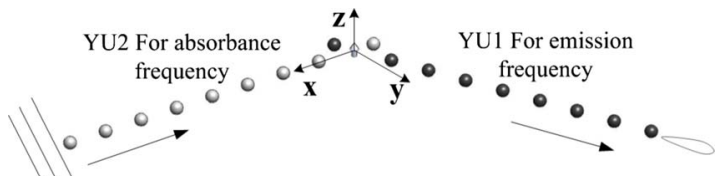

(a)

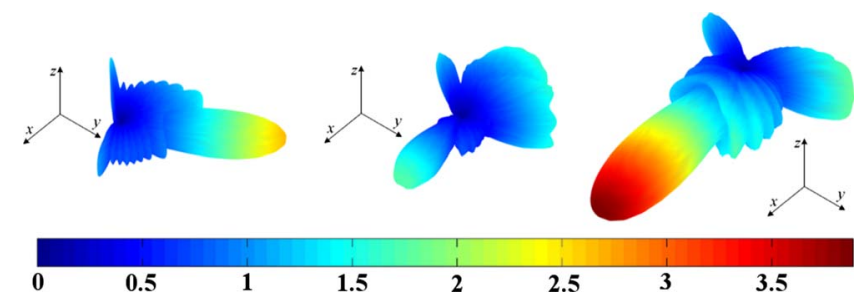

(b)

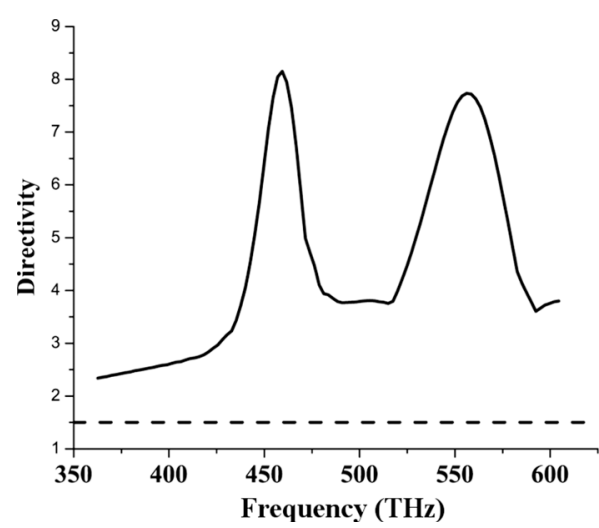

(c)

FIG. 5. (Color online) Optical nanoscale spectrometer with noncollinear configuration. (a) The geometry of the system. (b) The radiated power patterns of the system at different operating wavelengths, up: at $464 \mathrm{THz}(646 \mathrm{~nm})$, middle: at $518 \mathrm{THz}(579 \mathrm{~nm})$, low: at $561 \mathrm{THz}$ $(534 \mathrm{~nm})$, and should be viewed as a receiving pattern when YU2 is at the receiving mode. (c) The directivity. 
are separated away not only in terms of wavelengths, but also in the (spatial) angular domain. Moreover, for the emission spectrum, we may use several optical nanoantennas each designed for a certain wavelength and each oriented along a certain direction around the fluorescent molecule. This collection of nanoparticles would provide the possibility of analyzing the spectrum of emission, transforming the spectral information into the angular information in term of a "rainbow" around the molecule. This may provide an alternative method for biosensing and nanotagging of molecules, providing different rainbows for different fluorescent molecules.

In the above discussion the optical Yagi-Uda nanoantenna proposed by us in Ref. 21 is used as an example of a building block to construct the spectrum analyzer presented here. However, other designs of optical nanoantennas are also pos- sible for this purpose if they provide good frequency and angular selectivity. For example, optical Yagi-Uda antennas with gold or silver nanorods of well-designed length as the passive elements ${ }^{17}$ may also be a good candidate for the spectrum analyzer discussed here. Using an extra antenna element (such as a nanorod with a gap) placed in proximity of the dipole source (i.e., close to the molecule) may further enhance the emission. ${ }^{26,27}$ All these are of great interests for future studies.

\section{ACKNOWLEDGMENTS}

This work is supported in part by the U.S. Air Force Office of Scientific Research (AFOSR) under Grant No. FA9550-08-1-0220.

\footnotetext{
*Author to whom correspondence should be addressed; engheta@ee.upenn.edu

${ }^{1}$ K. B. Crozier, A. Sundaramurthy, G. S. Kino, and C. F. Quate, J. Appl. Phys. 94, 4632 (2003).

${ }^{2}$ V. A. Podolskiy, A. K. Sarychev, E. E. Narimanov, and V. M. Shalaev, J. Opt. A, Pure Appl. Opt. 7, S32 (2005).

${ }^{3}$ P. Muhlschlegel, H. J. Eisler, O. J. F. Martin, B. Hecht, and D. W. Pohl, Science 308, 1607 (2005).

${ }^{4}$ J. Li and N. Engheta, IEEE Trans. Antennas Propag. 55, 3018 (2007).

${ }^{5}$ A. Alu and N. Engheta, Phys. Rev. Lett. 101, 043901 (2008).

${ }^{6}$ A. Alu and N. Engheta, Nat. Photonics 2, 307 (2008).

${ }^{7}$ R. R. Chance, A. Prock, and R. Silbey, Advances in Chemical Physics, Vol. 37, edited by I. Prigogine and S. A. Rice (John Wiley \& Sons, New York, 1978), pp. 1-65.

${ }^{8}$ W. L. Barnes, J. Mod. Opt. 45, 661 (1998).

${ }^{9}$ R. L. Hartman, J. Opt. Soc. Am. A Opt. Image Sci. Vis. 17, 1067 (2000).

${ }^{10}$ N. Danz, R. Waldhausl, and A. Brauer, J. Opt. Soc. Am. B 19, 412 (2002).

${ }^{11}$ J. Azoulay, A. Debarre, A. Richard, and P. Tchenio, Europhys. Lett. 51, 374 (2000).

${ }^{12}$ H. Chew, Phys. Rev. A 38, 3410 (1988).

${ }^{13}$ R. R. Chance, A. Prock, and R. Silbey, J. Chem. Phys. 60, 2184 (1974).
}

${ }^{14}$ M. Thomas, J. J. Greffet, R. Carminati, and J. R. Arias-Gonzalez, Appl. Phys. Lett. 85, 3863 (2004).

${ }^{15}$ H. Gersen, M. F. Garcia-Parajo, L. Novotny, J. A. Veerman, L. Kuipers, and N. F. van Hulst, Phys. Rev. Lett. 85, 5312 (2000).

${ }^{16}$ A. L. Mattheyses and D. Axelrod, J. Biomed. Opt. 10, 054007 (2005).

${ }^{17}$ H. F. Hofman, T. Kosako, and Y. Kadoya, New J. Phys. 9, 217 (2007).

${ }^{18}$ T. H. Taminiau, F. D. Stefani, and N. F. van Hulst, Opt. Express 16, 10858 (2008).

${ }^{19}$ R. de Waele, A. F. Koenderink, and A. Polman, Nano Lett. 7, 2004 (2007).

${ }^{20}$ T. H. Taminiau, F. D. Stefani, F. B. Segerink, and N. F. van Hulst, Nat. Photonics 2, 234 (2008).

${ }^{21}$ J. Li, A. Salandrino, and N. Engheta, Phys. Rev. B 76, 245403 (2007).

${ }^{22}$ N. Halas, MRS Bull. 30, 362 (2005).

${ }^{23}$ H. Yagi, Proc. IRE 16, 715 (1928).

${ }^{24}$ J. D. Kraus and R. J. Marhefka, Antennas for All Applications, 3rd ed. (McGraw-Hill, New York, 2002).

${ }^{25}$ P. B. Johnson and R. W. Christy, Phys. Rev. B 6, 4370 (1972).

${ }^{26}$ P. Anger, P. Bharadwaj, and L. Novotny, Phys. Rev. Lett. 96, 113002 (2006).

${ }^{27}$ S. Kühn, U. Håkanson, L. Rogobete, and V. Sandoghdar, Phys. Rev. Lett. 97, 017402 (2006). 